\title{
Estudios feministas de religión: una mirada muy parcial"
}

\author{
Mónica Tarducci** \\ la Biblia y la Iglesia han sido el escollo más grande \\ en el camino de la emancipación de las mujeres \\ Elizabeth Cady Stanton (siglo XIX)
}

\section{Antecedentes}

Así como el feminismo como movimiento social nació en los países protestantes, los estudios sobre religión desde su perspectiva son producidos mayoritariamente en ellos, especialmente en los Estados Unidos.

Tanto quienes buscan los orígenes de la Teología Feminista, como quienes historicizan la relación feminismo-religión no pueden dejar de mencionar a The Woman's Bible de Elisabeth Cady Stanton, cuyo primer volumen fue publicado en 1895. Stanton, activa militante sufragista, relacionaba en sus artículos y conferencias la posición subordinada de las mujeres en el ámbito del poder de la iglesia, criticando radicalmente el uso de las Escrituras en favor de la esclavitud, de la pena de muerte y en contra de la obtención de los derechos ciudadanos de las mujeres.

El proyecto de Stanton fue revisar críticamente tanto los contenidos sexistas de la Biblia como la deliberada ausencia de las mujeres en distintos pasajes de ésta. Fue una obra colectiva, que involucró a treinta mujeres de Estados Unidos y Europa,

* Recebido para publicação em outubro de 2001.

** Antropóloga. Instituto Interdisciplinario de Estudios de Género. Facultad de Filosofía y Letras, Universidad de Buenos Aires. Profesora de la Maestría de Género de la Universidad de Rosario. tarducci@fibertel.com.ar 
Estudios feministas de religión

especialistas en religión y en lenguas antiguas y que provocó muchas polémicas en el movimiento feminista del momento, al punto que la National American Women Suffrage Association, de la cual Stanton era cofundadora, rechazó cualquier conexión con la obra, temerosa de que los asuntos religiosos trajeran divisiones entre las mujeres.

Durante años la religión, vista como una fuente de subordinación de las mujeres, fue un tema intratable para el movimiento feminista. Una relación ambivalente, ya que los temas que interesan a la religión son parte de la agenda feminista: sexualidad, reproducción, parentesco, aborto.

En 1960 se produce un hito en la teología feminista: la publicación de The human situation: a feminine view de Valerie Saiving, en el Journal of Religion del mes de abril. La autora critica la teología desde la perspectiva de la "experiencia femenina" antes de comenzar la segunda ola del movimiento feminista, afirmando que la visión de los teólogos está afectada por las particularidades de su experiencia como varones o como mujeres. Revolucionariamente comienza su artículo diciendo "Soy una estudiante de teología y también soy una mujer".

\section{El pecado original}

Todas conocemos el impacto que el movimiento feminista ha tenido en las Ciencias Sociales en los últimos treinta años. Si bien los Estudios de Religión no estuvieron al margen de esas influencias, creo que la relación ha sido más conflictiva, por el propio origen de éstos. En efecto, su "pecado original" fue haber nacido como estudios teológicos.

Según Carol Christ, ${ }^{1}$ antes de 1960 , la religión se enseñaba fundamentalmente desde una perspectiva de las distintas denominaciones. Las universidades más importantes tenían

${ }^{1}$ CHRIST, Carol. Toward a Paradigm Shift in the Academy and in Religious Studies. In: FARNHAM, C. (ed.) The impact of Feminist Research in the Academy. Bloomington, Indiana University Press, 1987, p.61. 
seminarios o divinity schools para formar a los pastores protestantes y clérigos católicos y judíos. También se ofrecían maestrías o doctorados en teología

La Religious Research Association y la Society for the Scientific Study of Religion celebraron juntas su cincuentenário en Boston, entre el 5 y el 7 de noviembre de 1999. Es interesante leer en el número especial del Journal for the Scientific Study of Religion dedicado a este acontecimiento ${ }^{2}$ el detallado análisis de la historia de las dos más importantes entidades académicas consagradas al estudio de los fenómenos religiosos. Se señala que ambas organizaciones se establecieron gracias al esfuerzo de las principales denominaciones protestantes y quienes enseñaban allí usaron los métodos y herramientas de la sociología para servir a las agencias denominacionales y ecuménicas. Claramente se veía un interés de las iglesias en la descripción y análisis del contexto social en el que ellas estaban insertas y rechazaban los análisis de la religión per se.

A partir de los años sesenta ${ }^{3}$, la influencia de la metodología sociológica ha permitido el deslizamiento, no sin conflictos, desde una "sociología pastoral" hacia una sociología de la religión. En cuanto a las temáticas abordadas, ese deslizamiento se proyectó hacia la "descristianización," lo que redundó en posiciones menos etnocéntricas, ya que, salvo los estudios llevados a cabo desde la perspectiva antropológica, "la religión" sólo era pensada dentro de los límites cristianos o, a lo sumo, judíos. También es notable la incorporación de los Nuevos Movimientos Religiosos como un objeto de análisis legítimo dejando de lado su estigmatizada visión como "sectas".

Metodológicamente se vio también un acercamiento hacia los análisis cualitativos como consecuencia del llamado cultural turn en las Ciencias Sociales, que se evidencia en la atención puesta en la dinámica de los sistemas de significado, en los

2 Journal for the Scientific Study of Religion, vol. 39, n 4, december 2000.

3 Tener en cuenta la influencia del Concilio Vaticano II. 
Estudios feministas de religión

símbolos y rituales, las prácticas devocionales, los fenómenos de conversión, de búsqueda espiritual. La perspectiva antropológica invade con su visión a micro-nivel las grandes síntesis sociológicas.

Dentro de esta apertura de los Estudios de Religión, y paralela a la politización de muchas pensadoras creyentes, hacen su entrada artículos claramente situados en una perspectiva feminista. De una crítica al androcentrismo de la disciplina y de las concepciones judío-cristianas acerca de las mujeres ${ }^{4}$, propio de las primeras épocas, se llega a la utilización de la categoría género como herramienta analítica, como proceso activo que articula múltiples aspectos de la vida social, entre ellos la experiencia religiosa que, como dice Caroline Walker Bynum, "es la experiencia de los hombres y las mujeres, y en ninguna sociedad conocida esa experiencia es la misma". ${ }^{5}$

Pero esa diferencia no es neutral, sino que opera dentro de sociedades y culturas que establecen relaciones jerárquicas de los hombres sobre las mujeres. Es por eso que si bien "género" incluye la construcción cultural de la masculinidad, la mayoría de los trabajos sobre religión y género tratan el universo de las mujeres en un intento de balancear la trayectoria androcéntrica de la disciplina.

Al igual que en otros campos del conocimiento, la "irrupción de las mujeres" ha redefinido críticamente las áreas de interés, creado modos de aproximación diferentes, y planteando nuevos interrogantes: ¿cuáles son los roles y el estatus de las mujeres en las tradiciones e instituciones religiosas?; ¿cómo son las representaciones de las mujeres en el lenguaje y el pensamiento religioso?; ¿qué relación existe entre la representación simbólica de las mujeres y su impacto sobre la vida cotidiana?; ¿cómo

4 The Church and the Second Sex, de Mary Daly, publicado en 1968, es considerada la primera crítica feminista del campo católico.

${ }^{5}$ Bynum, Caroline Walker. Introduction: The Complexity of Symbols. En ByNum, C.W.; Harrell, S. y Richman, P. (eds.) Gender and Religion: On the Complexity of Symbols. Boston, Beacon Press, 1986, p.2. 
podemos describir e interpretar la experiencia religiosa de las mujeres?.

Metodológicamente, no sólo se deconstruye el marco androcéntrico sino que también se reconstruye la experiencia de las mujeres, tratando de comprender lo que recibió poca atención, lo que no ha sido escuchado o lo que ha sido interpretado de manera sesgada. ${ }^{6}$

Sin embargo, cuando comencé a interesarme por el tema, me sucedió (y me sigue sucediendo) que la gran mayoría de la bibliografía, listas de correo electrónico y grupos tengan una orientación teológica, y pareciera que ser feminista y preocuparse con la religión significa que se deba pertenecer a algún credo, sea de "las grandes religiones" o de algunas de las variantes de ese amplio movimiento llamado "Iglesia-Mujer", Teología Feminista, o "Espiritualidad feminista", área ésta donde más influencia han tenido las ideas feministas.

Así, los Estudios de Religión (incluidos los feministas) es un área emocionalmente sesgada donde el o la investigador/a está comprometido con algún sistema de creencias que rara vez es explícito. Para alguien agnóstica como yo, y que comparte los objetivos políticos del feminismo, comprender una religión, tener empatía con el objeto de estudio, no es lo mismo que compartir cierta indulgencia de los estudios de religión con las facetas más oscuras de ésta, indulgencia que en general está basada en la valoración de éticas particulares producto de un relativismo cultural culposo.

Por otro lado, si bien como feminista comparto muchos espacios con teólogas y mujeres sinceramente involucradas en la espiritualidad feminista, posición que respeto y valoro, como investigadora me planteo el problema de que la mayoría de sus trabajos no dan cuenta de cuál es el impacto de las religiones

${ }^{6}$ KING, Ursula. Introduction: Gender and the Study of Religion. En KING, U. (ed.) Religion and Gender. Oxford, Cambridge University Press, 1995. 
Estudios feministas de religión

existentes en el mundo actual, sobre la gran mayoría de las mujeres.

\section{El enfoque comparativo}

Después de treinta años de desarrollo de una perspectiva de género en los estudios de religión, se han hecho algunos avances que tratan de ir más allá de los casos particulares para intentar, de manera provisoria, de proveernos de algunas sugerencias de carácter comparativo que nos ayuden a comprender mejor ciertas áreas de esta disciplina, sin olvidar, por supuesto, que las generalizaciones muy amplias tienden a minimizar las variaciones internas de cualquier tradición religiosa.

Rita Gross ${ }^{7}$, citando un trabajo anterior de Katherine Young, traza un panorama del mundo de las religiones a lo largo de un continuum que se mueve desde un mayor predominio (formal o proclamado) masculino hacia una mayor aceptación o inclusión del poder femenino. Sugiere el siguiente orden: judaísmo, confucionismo, islán, hinduismo, cristianismo, budismo y taoísmo.

Luego establece otros criterios, donde las religiones pueden cambiar su posición en el continuum. Si separamos a las religiones en aquellas que están orientadas principalmente hacia unidades familiares y sociales y aquellas que lo están hacia los individuos, vemos que el confucianismo, el judaísmo y algunas versiones del hinduismo fundamentalmente se orientan hacia la familia y la sociedad, más que hacia el individuo. Las religiones que están orientadas hacia la familia por lo general tienen códigos de conducta y expectativas específicos para cada sexo y públicamente favorecen el dominio masculino. Usualmente las mujeres son confinadas a los roles domésticos y familiares y no participan de manera significativa en las actividades religiosas fuera del hogar. Hasta hace poco tiempo la educación religiosa formal era una excepción y las mujeres casi nunca ejercían, de

7 Gross, Rita. Feminism and Religion. Boston, Beacon Press, 1996. 
manera pública, un liderazgo político o religioso. Existe una preferencia cultural por la masculinidad y la descendencia masculina se considera esencial para el bienestar de la familia y en consecuencia los niños son más bien cuidados que las niñas, tanto física como psicológicamente. Como la familia ocupa un lugar tan central, los roles familiares de las mujeres son abrumadoramente importantes.

El cristianismo, el budismo, el taoísmo y algunos segmentos del hinduismo están menos centrados en la familia y más involucrados con el bienestar espiritual de los individuos. Especialmente las tres primeras tienen instituciones monásticas donde las mujeres han participado. $O$ sea que existe una alternativa a los roles familiares y de alguna manera $\mathrm{u}$ otra, preconizan ciertos principios de equidad sexual. Como son estructuras fuertemente jerarquizadas y de dominación masculina, el acceso a las instancias de decisión y a ciertas dimensiones de la vida espiritual es frecuentemente limitado. Por otra parte existen tendencias misóginas que se manifiestan en la sobrevaloración del ascetismo y del celibato. Especialmente en el cristianismo y en el budismo, la mujer es vista como menos espiritual y más pecadora que los hombres.

Por último, la tercera clasificación de Gross toma un tema caro a algunos sectores del feminismo: la presencia o ausencia de deidades femeninas en las religiones examinadas. Si bien las religiones monoteístas no tienen oficialmente diosas, nunca han logrado desprenderse de los aspectos femeninos de la divinidad. En el resto de las religiones del mundo las deidades son un lugar común del mundo religioso. En este continuun, islán, judaismo, cristianismo, taoismo, budismo, shintoismo e hinduismo se mueven desde la ausencia a la presencia de deidades femeninas. El hecho de que religiones de fuerte dominio masculino tengan diosas socava la correlación establecida entre presencia de ellas y posición alta de las mujeres. 
Estudios feministas de religión

\section{Creer con el cuerpo}

Una de las áreas más fructíferas de los estudios feministas de la religión tiene que ver la atención puesta en los últimos años en la relación entre cuerpo y religión.

Los trabajos que se ocupaban del cuerpo en relación con la religión, se centraban en general sobre las ideas acerca del cuerpo, especialmente los que se refieren al peso que tiene la concepción de éste en occidente. En efecto, al menos desde la época clásica y el cristianismo, el cuerpo es visto como un fenómeno peligroso y amenazante si no se lo controla y regula por un proceso cultural. Es un vehículo o recipiente de lo ingobernable, las pasiones irracionales, los deseos y las emociones.

Muchas pensadoras feministas han resaltado la relación entre ideologías dualistas, que promueven la des-corporalidad y la trascendencia y la devaluación de los cuerpos, especialmente el cuerpo femenino. Por otro lado, los mitos acerca de la naturaleza sexual de las mujeres están presente no sólo en occidente: la menstruación y el parto en general son vías de polución tanto en el hinduismo (no pueden acercarse al santuario familiar durante la menstruación y el embarazo), ni las musulmanas pueden tocar el Corán durante la menstruación, ni orar ni entrar a la mezquita) y lo mismo sucede con muchas sociedades estudiadas por la antropología.

Sin embargo, en los últimos años, se ha comenzado a llamar la atención acerca de que el conocimiento del cuerpo es central en el estudio de la religión porque, entre otras cosas, muchas de las experiencias corporales, incluida la propia fisiología, son percibidas y expresadas religiosamente. El cuerpo es construido, desmembrado o reparado en el ritual. Los cambios del ciclo vital: nacimiento, desarrollo, muerte, polución, purificación, son a menudo momentos claves de la acción simbólica comunal. Los sentidos son reorientados y las percepciones del cuerpo son 
Mónica Tarducci

corregidas o reordenadas a través del contacto ritual con lo sagrado. ${ }^{8}$

La importancia del cuerpo en los estudios de religión abre un amplio panorama para la antropología, no sólo respecto del fenómeno ritual, sino también en la comprensión de cómo la religión está vinculada a las emociones, los sentimientos, las percepciones, la imaginación y la memoria. ${ }^{9}$

Hoy hay mayor conciencia de la importancia de la emoción como corporizada, como un aspecto de la experiencia corporal y no sólo en las religiones marginales. Prestando atención a conceptos como embodiment y performance se estudian tácticas somáticas como "armas del débil": brujería y hechicería y su contra-cara; trance y posesión; ataques de nervios y locura; rituales de inversión y juegos de fantasía, donde el caos y la liminaridad se imponen, trastocando el orden de una vida cotidiana opresiva.

La posesión sirve a una variedad de propósitos para las poseídas. En primer lugar libera a las mujeres de la culpa asociada a la rebelión y también de su responsabilidad por la conducta antisocial. Pueden decir y hacer cosas que nunca se les permitirían si tuvieran el control de sí mismas. Donde las estructuras religiosas no son tan rígidas o controladas, la posesión brinda a las mujeres acceso a un poder real en el culto. Muchas mujeres poseídas han creído tener el suficiente control sobre los espíritus como para tener la habilidad de trabajar en la cura, la adivinación y otros poderes. Tales mujeres son particularmente útiles en curar a otras con aflicciones similares.

8 SulLIVAN, Lawrence. Body Works: Knowledge of the body in the study of religion. History of Religion, vol. 30, n 1, 1990, pp.86-99.

9 MCGUIRE, Meredith. Religion and the body: Rematerializing the human body in the social sciences of religion. Journal for the Scientific Study of Religion, 29 (3), 1990, pp.283-296. 
Estudios feministas de religión

Anne Ong ${ }^{10}$ estudió los casos de posesión entre las obreras de factorías multinacionales de Malasia, quienes se transforman en seres violentos como protesta ante la rigidez de la rutina del trabajo, y su devaluación como personas dentro y fuera de la fábrica. Esos desórdenes periódicos son racionalizados como psiquiátricos, reforzando así la lógica del capitalismo global y cómo esto se entrecruza con las creencias locales produciendo la respuesta de la posesión.

\section{Ministerio}

Es indudable que las reivindicaciones feministas han presionado, y lo siguen haciendo, por una apertura de las instituciones religiosas que permita el ministerio de las mujeres. Solo recientemente algunas denominaciones protestantes y algunos sectores del judaísmo aceptan a las mujeres como clérigas.

La afirmación general de que las mujeres son marginales en las iglesias hace referencia a varias situaciones: a que no se les permite la ordenación, a que sí se les permite, pero no de manera completa, así como a quienes son ordenadas con pleno derecho y buscan cambiar la naturaleza del ministerio. ${ }^{11}$

Pero también las mujeres asumen un lugar mucho más importante, tanto en el liderazgo como en la participación, en las religiones populares y disidentes más que en las establecidas, se vean o no a si mismas como opositoras a la autoridad establecida. Las mujeres tienden a surgir como ministras en los movimientos religiosos que no realizan un control sobre su clerecía y que dependen de una emoción religiosa auténtica y espontánea. Por eso, los grupos evangélicos de organización más laxa tienen frecuentemente ministras mujeres.

${ }^{10}$ ONG, Anne. The production of possession: spirits and the multinational corporation in Malaysia. American Ethnologist 15, 1988, pp.28-44.

${ }^{11}$ WaLlaCE, Ruth. Bringing women in. Sociological Analysis 50, pp.291-303. 
Mary F. Bednarowsky, en 1980, discutía los factores comunes que se pueden encontrar las religiones con mujeres en roles de liderazgo importantes. ${ }^{12}$

$\mathrm{Si}$ bien las mujeres eran fundamentalmente el mayor número de creyentes, en las principales denominaciones los teólogos, ministros y líderes eran hombres. Bednarowsky propuso que el liderazgo femenino se da en religiones que poseen las siguientes cuatro características:

1. una percepción de lo divino que des-enfatiza lo masculino, tanto en el sentido de una divinidad bisexual, impersonal o no antropomórfica;

2. una atenuación o rechazo a la doctrina de la caída;

3. una renuncia a la ordenación tradicional de clérigos;

4. una visión del matrimonio que no enfatice a éste y a la maternidad como la esfera apropiada para las mujeres y sus únicas fuentes de realización.

La participación mas activa de las mujeres como líderes religiosas la tenemos que buscar en las llamadas religiones "marginales": grupos que se separan voluntariamente del mainstream para preservarse de lo que consideran un mundo pecaminoso (algunos pentecostales); grupos que no se aíslan del orden social pero rechazan la religiosidad de sociedad (espiritualismo, teosofía, Nuevo Pensamiento, espiritualidad feminista); grupos que se sienten excluidos por el racismo (diferentes religiosidades afro-americanas); grupos que se sienten excluidos por el sexismo (movimiento Iglesia-Mujer) y grupos que resultan de la importación de ideas religiosas (budistas, hinduistas). ${ }^{13}$

\footnotetext{
${ }^{12}$ BednAROWSKI, Mary Farrell. Outside the Mainstream: Women's Religion and Women Religious Leaders in Nineteenth-Century America. En Journal of the American Academy of Religion 48, june 1980, pp.207-231.

${ }^{13}$ Wessinger, Catherine. Going Beyond and Retaining Charisma: Women's Leadership in Marginal Religions. En: WeSsINGER, C. (ed.) Women's Leadership in Marginal Religions. Chicago, University of Illinois Press, 1993.
} 
Estudios feministas de religión

En interesante el caso de las pastoras pentecostales, ya que existe una paradoja entre sus deseos de liderazgo, que deben ser legitimados con una retórica que enfatiza su subordinación. La continua tensión entre la feminidad pentecostal y sus roles pastorales, deja poco espacio para trabajar a favor de la equidad de género.

Edward Lehman, que condujo una investigación empírica sobre mujeres y hombres clérigos en las iglesias protestantes históricas, a lo largo de diez años, se centró en los "estilos" de ministerio de varones y mujeres, situándose a distancia de las posiciones (que denomina maximalistas) de quienes afirman que las mujeres llevan a los grupos religiosos la incorporación de relaciones holísticas, igualitarismo, empowerment de los miembros de la congregación, tomas de decisión democráticas, una teología mas abierta y flexible, mayor responsabilidad, entre otras cosas. En cambio, para los "minimalistas", las diferencias entre hombres y mujeres en el ministerio, no son rasgos específicos de personalidad basados en la diferencia sexual, sino epifenómenos del lugar que ocupa el género en la estructura social. ${ }^{14}$

¿Cuales son las conclusiones a las que arriba Lehman? Dados los resultados de la investigación, que con mucho humor el autor resume en la frase "eso depende" y, aclarando que, si preguntamos inocentemente "ilos hombres y las mujeres tienen un estilo de ministerio diferente?", la respuesta siempre es afirmativa, afirma que lo correcto sería preguntar "¿bajo qué condiciones las mujeres y los hombres ejercen su ministerio de manera diferente"? y llega a algunas conclusiones que él denomina empíricas.

1. Existen algunas diferencias específicas por género entre hombres y mujeres pastores. Más de lo que los minimalistas suponen y menos claras de lo que los

\footnotetext{
${ }^{14}$ LeHMAN, Edward. Gender and Ministry Style: Things Are Not What They Seem. En SwATOS, W. (ed.) Gender and Religion. New Brunswick-Londres, Transaction, 1994, pp.3-26.
} 
maximalistas proponen. Depende de que dimensiones se tengan en cuenta;

2. La fuerza de esas diferencias tiende a suavizarse, sugiriendo que las diferencias en los estilos de ministerio no son tan grandes como se había argumentado;

3. Cuando esas diferencias de estilo aparecen, dependen de condiciones biográficas, culturales y estructurales identificables.

\section{Fundamentalismo}

Como en ningún otro campo de los estudios feministas de religión, el fundamentalismo, por su complejidad, extensión espacial, rápido crecimiento, la cantidad de variables que pone en juego, las polémicas que genera, nos permite poner a prueba todo lo trabajado desde la perspectiva de género hasta ahora. La clave de muchas discusiones está en el cambio hacia la focalización de la agency humana en la teorización feminista. Muchas investigadoras han comenzado a ver a ésta ubicada dentro de estructuras de subordinación y tratan de comprender los modos en que las mujeres resisten el orden dominante masculino subvirtiendo los significados hegemónicos de las prácticas culturales y re-utilizándolas para sus propios intereses y agendas.

En 1987, Fátima Mernissi, con un conocimiento profundo de Egipto en particular y de los países musulmanes en general, analizó los cambios demográficos, políticos, educacionales, laborales para poder explicar la situación de las mujeres dentro del contexto de auge del fundamentalismo. "La ola conservadora contra las mujeres en el mundo musulmán, lejos de ser una tendencia retrógrada, es, por el contrario, un mecanismo de defensa contra los profundos cambios en los roles de ambos sexos y en el tema tan susceptible de la identidad sexual." ${ }^{15}$ Este punto

\footnotetext{
${ }^{15}$ MeRnISSI, Fátima. Beyond the Veil. Male-Female Dynamics in Modern Muslim
} Society. Bloomington-Indianapolis, Indiana University Press, 1987, pp.xxvii. 
Estudios feministas de religión

de vista que sitúa al fundamentalismo como reacción antimoderna es general, compartido aún hoy, y para diferentes religiones, por autores tales como Lawrence, Reisebroth, entre otros. ${ }^{16}$

Desde la publicación de ese libro, mucho se ha escrito y la situación hoy es compleja y contradictoria. Lo que es imposible de soslayar es la importancia de una perspectiva de género, que arroja una luz reveladora sobre la naturaleza del fundamentalismo en general, que demostraría ciertos "aires de familia" en sus distintas manifestaciones, más allá de la diversidad. Ese aire de familia serían sus concepciones acerca de la familia, las relaciones interpersonales, la sexualidad, los roles sexuales, la educación de los niños, y de ahi se derivan reglas que afectan la vida cotidiana de todas las personas involucradas, aunque su peso recae especialmente sobre las mujeres. ${ }^{17}$

Martin Marty y R. Scott Appleby dirigen desde 1988 el Fundamentalism Project, en la Universidad de Chicago. Este estudio sistemático y transcultural del fundamentalismo lleva editado varios volúmenes. El segundo, de 1993, Fundamentalism and Society, toma en consideración cuestiones de género. ${ }^{18}$

Otra obra digna de destacar es Mixed Blessings, ${ }^{19}$ donde se presenta un panorama del fundamentalismo en diferentes religiones, agrupando los artículos en tres ítems: a) mujeres que se benefician del fundamentalismo; b) mujeres que luchan por ganar poder y autonomía dentro de las limitaciones del fundamentalismo y c) mujeres oprimidas por el fundamentalismo.

\footnotetext{
${ }^{16}$ Véase HAWLEY, John Stratton. (comp.) Fundamentalism and Gender. Nueva York-Oxford: Oxford University Press, 1994.

${ }^{17}$ TARDUCCI, Mónica. Fundamentalismo y relaciones de género: "aires de familia" más allá de la diversidad. Ciencias Sociales y Religión/Ciencias Sociais e Religiao, año $1, \mathrm{n}^{\circ} 1$, setembro 1999, pp.189-211.

${ }^{18}$ HARDACRE, Helen. The impact of fundamentalisms on women, the family, and interpersonal relations. En: MARTY, M. y APPLEBY, S. (comp.) Fundamentalisms and Society. Chicago-Londres, University of Chicago Press, 1993, pp.129-150.

${ }^{19}$ Brink, Judy and MENCHER, Joan. Mixed Blessings. Gender and Religious Fundamentalism Cross Culturally. Nueva York, Routledge, 1997.
} 
Publicado en 1997, es una puesta al día de la problemática del fundamentalismo y una discusión con obras anteriores, como la ya citada compilación de Marty y Appleby y con Fundamentalism and Gender, editada por John S. Hawley. ${ }^{20}$

Las compiladoras caracterizan a los artículos fuera de lo que ellas consideran una posición desde del punto de vista androcéntrico, señalando varias cuestiones para poder dar cuenta de la contradicción: "si es un movimiento masculino por excelencia, ¿̇por qué tantas mujeres se involucran en propuestas fundamentalistas?". El amplio panorama presentado, que recorre el pentecostalismo en Colombia, las monjas budistas de Sri Lanka, las musulmanas de una región de China, de Pakistán, de Egipto y de Sudán, las evangélicas y mormonas de Estados Unidos, las judías de Palestina, así como la política fundamentalista en la India, permiten varias reflexiones y sugieren caminos a seguir. Entre ellos los siguientes:

1. La mayoría de los estudios sobre fundamentalismo tienen un enfoque masculino (aunque sean hechos por mujeres) $y$, a gran escala, focalizan más en los caracteres estructurales de los grupos y movimientos, en sus líderes que en el compromiso individual y en el trabajo etnográfico a pequeña escala. Se centran en la política de género a nivel del movimiento o del estado o en la retórica fundamentalista.

2. Mas que centrarse en la metáfora de la reacción, los artículos del libro prefieren emplear la metáfora de la agencia, esto es, utilizar las historias de vida y la etnografía, desde el punto de vista feminista, para comprender las circunstancias locales y los desafíos que plantea el fundamentalismo en la vida de las mujeres. Los matices de las negociaciones que llevan a cabo, mas allá de las reglas a las que son sometidas las mujeres.

${ }^{20}$ HaWley, J. S. (comp.) Fundamentalism and Gender. Op.cit. 
Estudios feministas de religión

3. Para ello es necesario prestar atención a las redes sociales, la vida familiar y las relaciones conyugales y el rol que juegan el ritual y la ideología religiosa, según las mujeres pertenezcan al ámbito rural o urbano, la pertenencia de clase o étnica, así como la importancia del estado o de eventos externos.

4. El grado de variación respecto del involucramiento y el grado de empowerment o sometimiento va a depender de circunstancias personales y contextuales. Los distintos trabajos exploran varias motivaciones personales de las mujeres: búsqueda de identidad, búsqueda de alivio en la vida personal o social, resistir el sexismo en el trabajo o compensar la carencia de opciones educacionales o de movilidad social.

5. No es suficiente estudiar los significados y el atractivo del fundamentalismo para las mujeres. Si afecta o no la autonomía de ellas, depende de factores políticos estructurales, de tradiciones preexistentes, factores personales, relaciones sociales y circunstancias económicas de las unidades domésticas. El impacto del mensaje puede alentar tanto la elevación de su situación como restricciones a la equidad.

6. Por último, existe una diferencia entre las mujeres que son afectadas por el fundamentalismo y aquellas que participan de manera entusiasta.

\section{Conclusiones}

Yo creo que el gran avance de los estudios feministas de la religión ha sido incorporar la experiencia de las mujeres a un conocimiento que se creía neutro. No sin rechazo, las investigadoras feministas han logrado un lugar, denunciando injusticias y explicando el origen y desarrollo de mucho sufrimiento, sometimiento y resignación. También mostrando el 
elusivo mundo de las mujeres mas allá de la retórica de la subordinación.

Ahora bien, noto en muchos de los artículos que leo un excesivo énfasis en ver los aspectos positivos de las prácticas religiosas como si hubiéramos pasado de considerar a la religión como una aberración, como un límite concreto a la libertad humana, que nos impone el "patriarcado" (el opio de los pueblos) a considerarla como un instrumento de afirmación cultural y política.

Por otro lado (y esto vale para los estudios de religión en general), si bien el cultural turn y los enfoques etnográficos iluminan áreas no tenidas en cuenta por las grandes síntesis, en estos modelos interpretativos de los fenómenos religiosos sucede que cualquier hipótesis sugerente a nivel comparativo es descalificada con las posibles excepciones a la regla. Algo que los antropólogos conocemos muy bien, y que en palabras de Mary Douglas adquieren el sarcástico nombre de "bongo-bonguismo": no podemos establecer tendencias generales sobre un fenómeno social porque siempre aparece un antropólogo que afirma que entre los "bongo-bongo" ese comportamiento no aparece.

Las investigadoras feministas ponen excesivo énfasis en los aspectos positivos de la agency y ven empowerment demasiado fácilmente. ¿A qué nos referimos cuando vemos el mejoramiento de la vida de las mujeres? ¿al nivel personal o al estructural? ¿basta con una auto-evaluación positiva de sus vidas? ¿qué criterios utilizamos? ¿ese bienestar personal lleva a las mujeres a cambios políticos y económicos de la sociedad en su conjunto?

Si leemos acerca del espiritualismo del siglo XIX en los Estados Unidos, por ejemplo, vemos que el empowerment de las mujeres las empujaba a la participación en asociaciones contra la esclavitud y por el sufragio femenino. Actualmente las distintas 
Estudios feministas de religión

manifestaciones de espiritualismo, en parte por su "individualismo radical", descartan el involucramiento en causas progresistas. ${ }^{21}$

Creo que, sin querer desvalorizar el esfuerzo de tantas estudiosas honestas, lo que percibo en los estudios feministas de religión es un voluntarismo que hace ver las cosas mejor de lo que están realmente, "elecciones" de las mujeres donde debería leerse "única opción", triunfos que son marginales a las duras, androcéntricas e invencibles jerarquías eclesiales que muy difícilmente se abren a la demanda de las mujeres y que harían bien en recordar las palabras de Mary Daly: "una mujer demandando equidad en la iglesia sería comparable a las personas negras demandando equidad en el Klu Klux Klan”.

${ }^{21}$ BRAUde, Ann. Radical Spirits: Spiritualism and Women's Rights in NineteethCentury America. Boston, Beacon Press, 1989. 